\title{
COHOMOLOGY OF HEISENBERG LIE ALGEBRAS
}

\author{
L. J. SANTHAROUBANE ${ }^{\prime}$
}

\begin{abstract}
The cohomology of Heisenberg Lie algebras is studied and we obtain the
\end{abstract} description of cocycles, coboundaries and cohomological spaces.

\section{Notations and preliminaries.}

1.1. Let $g$ be a Lie algebra of dimension $n$ over a field $F$ and $M$ a $g$-module of finite dimension over $F$. We denote by $C^{p}(g, M)$ the space of cochains of degree $p$, $d_{p}: C^{p}(g, M) \rightarrow C^{p+1}(g, M)$ the restriction to $C^{p}(g, M)$ of the coboundary operator, $Z^{p}(\underline{g}, M)$ the kernel of $d_{p}$ (space of cocycles of degree $\left.p\right), B^{p}(g, M)$ the range of $d_{p-1}$ (space of coboundaries of degree $\left.p\right), H^{p}(g, M)$ the quotient of $Z^{p}(g, M)$ by $B^{p}(g, M)$ (space of cohomology of degree $p$ of $g$ with values in $M$ ). If $M=F$, denote $C^{p}(g)=C^{p}(g, F), \quad Z^{p}(g)=Z^{p}(g, F), \quad B^{p}(g)=B^{p}(g, F), \quad H^{p}(g)=$ $H^{p}(g, F)$. For all details see $[1,2,5,6]$.

1.2. By the vector space isomorphisms

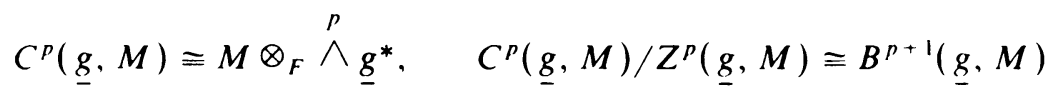

(where $\underline{g}^{*}$ is the dual of $\underline{g}$ and $\wedge^{p} \underline{g}^{*}$ the vector space of homogeneous elements of degree $\bar{p}$ of the Grassmann algebra over $\underline{g}^{*}$ ) one has

$$
\left(\begin{array}{l}
n \\
p
\end{array}\right) \operatorname{dim} M=\operatorname{dim} Z^{p}(\underline{g}, M)+\operatorname{dim} B^{p+1}(\underline{g}, M) ;
$$

therefore,

(i) $\operatorname{dim} H^{p}(\underline{g}, M)=\operatorname{dim} Z^{p}(\underline{g}, M)+\operatorname{dim} Z^{p-1}(\underline{g}, M)-\left(\begin{array}{c}n \\ p-1\end{array}\right) \operatorname{dim} M$,

(ii) $\operatorname{dim} H^{p}(\underline{g}, M)=\left(\begin{array}{l}n \\ p\end{array}\right) \operatorname{dim} M-\operatorname{dim} B^{p}(\underline{g}, M)-\operatorname{dim} B^{p+1}(\underline{g}, M)$.

Denote

$$
\chi_{p}(\underline{g}, M)=\sum_{q=0}^{p}(-1)^{q} \operatorname{dim} H^{q}(\underline{g}, M)
$$

Received by the editors January 30, 1982 and, in revised form, May 11, 1982.

1980 Mathematics Subject Classification. Primary 17B30, 17B56: Secondary 18G40, 18 G15.

Key words and phrases. Heisenberg Lie algebras, cohomology.

1 The author gratefully acknowledges the support of a grant from the French Government's "Délégation Gènérale à La Recherche Scientifique et Technique Paris" (Contract $n^{\circ}$ 77167). 
(partial sum of the Euler-Poincaré characteristic); by using $\sum_{q=0}^{p}(-1)^{q}\left(\begin{array}{c}n \\ q-1\end{array}\right)=$ $(-1)^{p}\left(\begin{array}{c}n-1 \\ p-1\end{array}\right)$ one obtains

$$
\begin{gathered}
\operatorname{dim} Z^{p}(\underline{g}, M)=(-1)^{p} \chi_{p}(\underline{g}, M)+\left(\begin{array}{c}
n-1 \\
p-1
\end{array}\right) \operatorname{dim} M, \\
\operatorname{dim} B^{p+1}(\underline{g}, M)=(-1)^{p} \chi_{p}(\underline{g}, M)+\left(\begin{array}{c}
n-1 \\
p
\end{array}\right) \operatorname{dim} M .
\end{gathered}
$$

(Remark. Since $C^{n+1}(g, M)=(0)$ one has $\operatorname{dim} B^{n+1}(g, M)=0$, therefore (iv) gives for the case $p=\bar{n}: 0=(-1)^{n} \chi_{n}(\underline{g}, M)+0 \cdot \operatorname{dim} M$; thus $\chi_{n}(\underline{g}, M)=0$. Goldberg obtains this result for $M=F[3]$.)

1.3. Let $g$ and $g^{\prime}$ be two Lie algebras of dimension $n$ and $n^{\prime}$ over $F, \rho$ and $\rho^{\prime}$ two representations of $\underline{g}$ and $\underline{g}^{\prime}$ into a vector space $M, \phi: \underline{g} \rightarrow \underline{g}^{\prime}$ a Lie algebra morphism such that $\rho=\rho^{\prime} \circ \bar{\phi}$. Denote

$$
\phi_{p}=(\stackrel{p}{\wedge} \phi)=\stackrel{p}{\wedge}^{\prime} \phi: C^{p}\left(\underline{g^{\prime}}, M\right) \rightarrow C^{p}(\underline{g}, M) ;
$$

then, obviously, $\phi_{p+1} \circ d_{p}^{\prime}=d_{p} \circ \phi_{p}$; since $\operatorname{Im} \phi_{p}=\wedge^{p}(\operatorname{Ker} \phi)^{\perp}$ it follows that

$$
\begin{aligned}
& \phi_{p}\left(Z^{p}\left(\underline{g}^{\prime}, M\right)\right) \subset \stackrel{p}{\wedge}(\operatorname{ker} \phi)^{\perp} \cap Z^{p}(\underline{g}, M), \\
& \phi_{p}\left(B^{p}\left(\underline{g}^{\prime}, M\right)\right) \subset \stackrel{p}{\wedge}(\operatorname{ker} \phi)^{\perp} \cap B^{p}(\underline{g}, M) .
\end{aligned}
$$

1.4. LEMMA. With the above notations, if $\phi$ is onto then

$$
\phi_{p}\left(Z^{p}\left(\underline{g^{\prime}}, M\right)\right)=\stackrel{p}{\wedge}(\operatorname{ker} \phi)^{\perp} \cap Z^{p}(\underline{g}, M),
$$

(ii) $\operatorname{dim} H^{p}\left(\underline{g}^{\prime}, M\right) \leqslant \operatorname{dim} H^{p}(\underline{g}, M)+\left(\left(\begin{array}{c}n \\ p-1\end{array}\right)-\left(\begin{array}{c}n^{\prime} \\ p-1\end{array}\right)\right) \operatorname{dim} M$.

$$
\operatorname{dim} H^{p}(\underline{g}, M) \leqslant \operatorname{dim} H^{p}\left(\underline{g}^{\prime}, M\right)+\left(\left(\begin{array}{l}
n \\
p
\end{array}\right)-\left(\begin{array}{c}
n^{\prime} \\
p
\end{array}\right)\right) \operatorname{dim} M .
$$

Proof. If $\phi$ is onto then $\phi_{p}$ is one-to-one; let $f \in \operatorname{Im} \phi_{p} \cap Z^{p}(g, M)$. One can write $f=\phi_{p} f^{\prime}$ with $f^{\prime} \in C^{p}\left(\underline{g}^{\prime}, M\right)$; then $0=d_{p} f=d_{p} \phi_{p} f^{\prime}=\phi_{p+1} d_{p}^{\prime} f^{\prime}$; therefore $d_{p}^{\prime} f^{\prime}=0$, i.e. $f^{\prime} \in Z^{p}\left(\underline{g}^{\prime}, M\right)$, which proves (i).

Since $\phi_{p}$ is one-to-one, we have

$$
\operatorname{dim} Z^{p}\left(\underline{g}^{\prime}, M\right)=\operatorname{dim} \phi_{p}\left(Z^{p}\left(\underline{g}^{\prime}, M\right)\right) \leqslant \operatorname{dim} Z^{p}(\underline{g}, M) ;
$$

thus

$$
\operatorname{dim} H^{p}\left(\underline{g}^{\prime}, M\right) \leqslant \operatorname{dim} Z^{p}(\underline{g}, M)+\operatorname{dim} Z^{p-1}(\underline{g}, M)-\left(\begin{array}{c}
n^{\prime} \\
p-1
\end{array}\right) \operatorname{dim} M
$$

(by 1.2(i)), which proves (ii) (by 1.2(i) again).

By considering $B^{p}$ instead of $Z^{p}$, (iii) is obtained in the same way.

1.5. RemarKs. (i) The inclusion $\phi_{p}\left(B^{p}\left(g^{\prime}, M\right)\right) \subset \wedge^{p}(\operatorname{ker} \phi)^{\perp} \cap B^{p}(\underline{g}, M)$ may be strict even with $\phi$ onto. For example let $\bar{g}=\mathfrak{Q}_{m}$ be the Heisenberg Lie algebra of dimension $2 m+1 \geqslant 5(2.2), \underline{g}^{\prime}=F e_{1}^{\prime} \oplus \cdots \oplus F e_{2 m}^{+}$the abelian Lie algebra of 
dimension $2 m$ and $\phi: \mathfrak{G}_{m} \rightarrow g^{\prime}, e_{i} \mapsto e_{i}^{\prime}, i \neq 0, e_{0} \mapsto 0$. One has (by (2.2))

$$
B^{p}\left(\mathfrak{Q}_{m}, M\right) \subset Z^{p}\left(\mathfrak{Q}_{m}, M\right)=\stackrel{p}{\wedge}\left(e^{* 1}, \ldots, e^{* 2 m}\right)=\stackrel{p}{\wedge}\left(F e_{0}\right)^{\perp}=\stackrel{p}{\wedge}(\operatorname{ker} \phi)^{\perp} ;
$$

thus

$$
B^{p}\left(\mathfrak{Q}_{m}, M\right) \cap \stackrel{p}{\wedge}(\operatorname{ker} \phi)^{\perp}=B^{p}\left(\mathfrak{Q}_{m}, M\right)
$$

since $\phi_{p}\left(B^{p}\left(g^{\prime}, M\right)\right)=\phi_{p}((0))=(0)$ and $B^{p}\left(\mathfrak{S}_{m}, M\right) \cong \wedge^{p}\left(e^{* 1}, \ldots, e^{* 2 m}\right)($ by $(2.2))$, the inclusion cannot be an equality.

(ii) For the above example of $\mathfrak{\mathcal { E }}_{m}$ and $g^{\prime}, 1.4($ ii) is an equality (by (2.2)). In low dimension there are several other examples realizing the equality and proving thus that the inequality cannot be improved.

\section{Cohomological spaces of Heisenberg Lie algebras.}

2.1. RemarK. By the Poincaré duality $H^{P}\left(\mathfrak{S}_{m}\right)$ and $H^{2 m+1-P}\left(\mathfrak{Q}_{m}\right)$ are canonically isomorphic; therefore, one has to study only $H^{p}\left(\mathfrak{Q}_{m}\right)$ for $p \leqslant m$.

2.2. THEOREM. Let $\mathfrak{S}_{m}=F_{0} \oplus \cdots \oplus F e_{2 m}$ be the Heisenberg Lie algebra of dimension $2 m+1$ over a commutative field $F$, i.e. a Lie algebra satisfying $\left[e_{i}, e_{i+m}\right]$ $=e_{0} \forall i=1, \ldots, m$ (all the other brackets are 0$)$. Let $p \in\{0, \ldots, m\}$ and denote by $\left\{e^{* 0}, \ldots, e^{* 2 m}\right\}$ the dual basis of $\left\{e_{0}, \ldots, e_{2 m}\right\}$. Then

(i) the pth Betti number (i.e. $\left.\operatorname{dim} H^{p}\left(\mathfrak{G}_{m}\right)\right)$ is equal to $\left(\begin{array}{c}2 m \\ p\end{array}\right)-\left(\begin{array}{c}2 m \\ p-2\end{array}\right)$;

(ii) the space of cocycles of degree $p$ of $\mathfrak{G}_{m}$ with values in $F$ is equal to the vector space of homogeneous elements of degree $p$ of the Grassmann algebra over $\left(e^{* 1}, \ldots, e^{* 2 m}\right)$, i.e.

$$
Z^{p}\left(\mathfrak{Q}_{m}\right)=\stackrel{p}{\wedge}\left(e^{* 1}, \ldots, e^{* 2 m}\right)
$$

(iii) the space of coboundaries of degree $p$ of $\mathfrak{S}_{m}$ with values in $F$ is isomorphic to the vector space of homogeneous elements of degree $p-2$ of the Grassmann algebra over $\left(e^{* 1}, \ldots, e^{* 2 m}\right)$, the isomorphism being given by the exterior product by $d_{1} e^{* 0}$ :

$$
\stackrel{p-2}{\wedge}\left(e^{* 1}, \ldots, e^{* 2 m}\right) \stackrel{\sim}{\rightarrow} B^{p}\left(\mathfrak{S}_{m}\right), \quad \gamma \mapsto d_{1} e^{* 0} \wedge \gamma .
$$

Proof. We will use induction on $m \geqslant 1$. The case $m=1$ is obvious.

Let $g^{\prime}=F e_{1}^{\prime} \oplus \cdots \oplus F e_{2 m}^{\prime}$ be the abelian Lie algebra of dimension $2 m$ and $\phi: \mathfrak{Q}_{m} \stackrel{g^{\prime}}{\rightarrow}$ the morphism defined by $\phi e_{i}=e_{i}^{\prime} \forall i=1, \ldots, 2 m, \phi e_{0}=0$. In the notation of 1.3 one takes $M=F, \rho=\rho^{\prime}=0$; then, by 1.4 (ii),

$$
\left(\begin{array}{c}
2 m \\
p
\end{array}\right) \leqslant \operatorname{dim} H^{p}\left(\mathfrak{E}_{m}\right)+\left(\begin{array}{c}
2 m+1 \\
p-1
\end{array}\right)-\left(\begin{array}{c}
2 m \\
p-1
\end{array}\right)
$$

and therefore $\left(\begin{array}{c}2 m \\ p\end{array}\right)-\left(\begin{array}{c}2 m \\ p-2\end{array}\right) \leqslant \operatorname{dim} H^{p}\left(\mathfrak{Q}_{m}\right)$.

Let $\mathfrak{Q}=F e_{0} \oplus \cdots \oplus F e_{2 m-1}$ and $\mathfrak{Q}_{m-1}=F e_{0} \oplus \cdots \oplus \widehat{F e}_{m} \oplus \cdots \oplus F e_{2 m-1}$ be the ideals of $\mathfrak{S}_{m}$ such that $\mathfrak{Q}=\mathfrak{Q}_{m-1} \times F e_{m}$ (direct product). By the theorem of 
Künneth [6, Chapter II, §5, p. 80] one has

$$
H^{p}\left(\mathfrak{Q}_{m-1} \times F e_{m}\right) \cong \sum_{i+j=p} H^{i}\left(\mathfrak{Q}_{m-1}\right) \otimes H^{j}\left(F e_{m}\right) ;
$$

therefore $\operatorname{dim} H^{p}(\mathfrak{S})=\operatorname{dim} H^{p}\left(\mathfrak{\mathscr { Q }}_{m-1}\right)+\operatorname{dim} H^{p-1}\left(\mathfrak{\mathscr { Q }}_{m-1}\right)$. Since $\mathfrak{Q}_{m-1}$ is a Heisenberg Lie algebra of dimension $2(m-1)+1$, the induction hypothesis gives $\operatorname{dim} H^{P}\left(\mathfrak{Q}_{m-1}\right)=\left(\begin{array}{c}2 m-2 \\ p\end{array}\right)-\left(\begin{array}{c}2 m-2 \\ p-2\end{array}\right)$ for $p \leqslant m-1$ and thus $\operatorname{dim} H^{p}(\mathfrak{Q})=\left(\begin{array}{c}2 m-1 \\ p\end{array}\right)$ $-\left(\begin{array}{c}2 m-1 \\ p-2\end{array}\right)$ for $p \leqslant m-1$. By Proposition 1 of [2] one has the following exact sequence (because $\mathfrak{G}$ is an ideal of codimension 1 in $\mathfrak{S}_{m}$ ):

$$
\cdots \rightarrow H^{p-1}(\mathfrak{Q}) \rightarrow H^{p}\left(\mathfrak{Q}_{m}\right) \rightarrow H^{p}(\mathfrak{Q}) \rightarrow H^{p}(\mathfrak{Q}) \rightarrow H^{p+1}\left(\mathfrak{Q}_{m}\right) \rightarrow \cdots
$$

which is a special case of the Hochschild-Serre spectral sequence [5]. Therefore, $\operatorname{dim} H^{p}\left(\mathfrak{S}_{m}\right) \leqslant \operatorname{dim} H^{p}(\mathfrak{Q})+\operatorname{dim} H^{p-1}(\mathfrak{Q})$ and thus $\operatorname{dim} H^{p}\left(\mathfrak{Q}_{m}\right) \leqslant\left(\begin{array}{c}2 m \\ p\end{array}\right)-\left(\begin{array}{c}2 m \\ p-2\end{array}\right)$ for $p \leqslant m-1$. Since the opposite inequality has already been proved one has conclusion (i) for $p \leqslant m-1$.

For $p=m$, one has to look more closely at the exact sequence of Dixmier. We proved that for $p \leqslant m-1$,

$$
\operatorname{dim} H^{p}\left(\mathfrak{S}_{m}\right)=\operatorname{dim} H^{p}(\mathfrak{S})+\operatorname{dim} H^{p-1}(\mathfrak{Q}) ;
$$

therefore, the long exact sequence splits into small exact sequences

$$
\begin{gathered}
(0) \rightarrow H^{0}\left(\mathfrak{Q}_{m}\right) \rightarrow H^{0}(\mathfrak{Q}) \rightarrow(0), \\
(0) \rightarrow H^{0}(\mathfrak{Q}) \rightarrow H^{1}\left(\mathfrak{Q}_{m}\right) \rightarrow H^{1}(\mathfrak{Q}) \rightarrow(0), \\
(0) \rightarrow H^{1}(\mathfrak{Q}) \rightarrow H^{2}\left(\mathfrak{Q}_{m}\right) \rightarrow H^{2}(\mathfrak{Q}) \rightarrow(0), \\
\cdots \cdots \cdots \cdots \\
(0) \rightarrow H^{m-2}(\mathfrak{Q}) \rightarrow H^{m-1}\left(\mathfrak{S}_{m}\right) \rightarrow H^{m-1}(\mathfrak{Q}) \rightarrow(0), \\
(0) \rightarrow H^{m-1}(\mathfrak{Q}) \rightarrow H^{m}\left(\mathfrak{S}_{m}\right) \rightarrow H^{m}(\mathfrak{Q}) \stackrel{u}{\rightarrow} H^{m}(\mathfrak{Q}) .
\end{gathered}
$$

One then has $\operatorname{dim} H^{m}\left(\mathfrak{S}_{m}\right)=\operatorname{dim} H^{m-1}(\mathfrak{Q})+\operatorname{dim} \operatorname{Ker} u$. Let $\alpha \in H^{m}\left(\mathfrak{Q}_{m-1}\right)$ and $\beta \in H^{m-1}\left(\mathfrak{\mathcal { E }}_{m-1}\right)$; one has

$$
\alpha \wedge \beta=c(\alpha, \beta) e^{* 0} \wedge \cdots \wedge \hat{e}^{* m} \wedge \cdots \wedge e^{* 2 m-1} \quad \text { with } c(\alpha, \beta) \in F .
$$

The duality of Poincare asserts that

$$
H^{m}\left(\mathfrak{Q}_{m-1}\right) \times H^{m-1}\left(\mathfrak{Q}_{m-1}\right) \rightarrow F, \quad(\alpha, \beta) \rightarrow c(\alpha, \beta)
$$

is a nondegenerate bilinear form; therefore $H^{m}\left(\mathfrak{Q}_{m-1}\right)$ is a quotient of

$$
\wedge^{m}\left(e^{* 0} \cdots \hat{e}^{* m} \cdots e^{* 2 m-1}\right)
$$

(since $H^{m-1}\left(\mathfrak{Q}_{m-1}\right)$ is a quotient of $\left.\wedge^{m-1}\left(e^{* 1} \cdots \hat{e}^{* m} \cdots e^{* 2 m-1}\right)\right)$. On the other hand, by the theorem of Künneth,

$$
H^{m}(\mathfrak{Q})=H^{m}\left(\mathfrak{S}_{m-1}\right)+H^{m-1}\left(\mathfrak{S}_{m-1}\right) \wedge e^{* m} .
$$


Now $u$ is the action of $e_{2 m}$ on $H^{m}(\mathfrak{Q})$ [2, Proposition 1]; since $\left[e_{m} e_{2 m}\right]=e_{0}$, $\left[e_{1} e_{2 m}\right]=0 . i \neq m$, one has $e_{2 m} \cdot e^{* 0}=e^{* m}, e_{2 m} \cdot e^{* i}=0, i \neq m$; therefore

$$
\operatorname{Im} u=\operatorname{Ker} u=H^{m-1}\left(\mathfrak{S}_{m-1}\right) \wedge e^{* m}
$$

thus

$$
\operatorname{dim} \operatorname{Ker} u=\operatorname{dim} H^{m-1}\left(\tilde{E}_{m-1}\right)=\left(\begin{array}{c}
2 m-2 \\
m-1
\end{array}\right)-\left(\begin{array}{c}
2 m-2 \\
m-3
\end{array}\right)
$$

(induction). We then have

$$
\begin{aligned}
\operatorname{dim} H^{m}\left(\tilde{\mathfrak{S}}_{m}\right) & =\left(\begin{array}{c}
2 m-1 \\
m-1
\end{array}\right)-\left(\begin{array}{c}
2 m-1 \\
m-3
\end{array}\right)+\left(\begin{array}{c}
2 m-2 \\
m-1
\end{array}\right)-\left(\begin{array}{c}
2 m-2 \\
m-3
\end{array}\right) \\
& =\left(\begin{array}{c}
2 m \\
m
\end{array}\right)-\left(\begin{array}{c}
2 m \\
m-2
\end{array}\right) .
\end{aligned}
$$

This proves conclusion (i) for $p=m$.

By a simple computation one has $(-1)^{p} \chi_{p}\left(\mathfrak{W}_{m}, F\right)=\left(\begin{array}{c}2 m \\ p\end{array}\right)-\left(\begin{array}{c}2 m \\ p-1\end{array}\right)$ and by 1.2(iii), another computation gives $\operatorname{dim} Z^{p}\left(\tilde{5}_{m}\right)=\left(\begin{array}{c}2 m \\ p\end{array}\right)$. From 1.3 it follows that $\phi_{p}\left(Z^{p}\left(g^{\prime}\right)\right) \subset Z^{p}\left(\tilde{\mathcal{D}}_{m}\right)$. Since $Z^{p}\left(\underline{g}^{\prime}\right)=\wedge^{p}\left(e^{\prime * 1}, \ldots, e^{\prime * 2 m}\right)$, we get $\phi_{p}\left(Z^{p}\left(\underline{g}^{\prime}\right)\right)=$ $\wedge^{p}\left(e^{* 1}, \ldots, e^{* 2 m}\right)$, therefore $\wedge^{\bar{p}}\left(e^{* 1}, \ldots, e^{* 2 m}\right) \subset Z^{p}\left(\mathfrak{S}_{m}\right)$. Conclusion (ii) then follows from $\operatorname{dim} Z^{p}\left(\tilde{\mathfrak{D}}_{m}\right)=\left(\begin{array}{c}2 m \\ p\end{array}\right)$.

Now

$$
B^{p}\left(\tilde{S}_{m}\right)=\left\{d_{p-1} \alpha ; \alpha \in \stackrel{p-1}{\wedge}\left(e^{* 0}, \ldots, e^{* 2 m}\right)\right\}
$$

Any $\alpha \in \wedge^{p-1}\left(e^{* 0}, \ldots, e^{* 2 m}\right)$ can be written $\alpha=\beta+e^{* 0} \wedge \gamma$ with $\beta \in$ $\wedge^{p-1}\left(e^{* 1}, \ldots, e^{* 2 m}\right) \quad\left(=Z^{p-1}\left(\mathfrak{S}_{m}\right)\right.$ by (ii)) and $\gamma \in \wedge^{p-2}\left(e^{* 1}, \ldots, e^{* 2 m}\right)$ ( $=Z^{p-2}\left(\mathscr{S}_{m}\right)$ by (ii)); thus $d_{p-1} \alpha=0+d_{1} e^{* 0} \wedge \gamma-e^{* 0} \wedge 0$ (recall that $d_{p}$ is an antiderivation); therefore

$$
B^{p}\left(\tilde{\mathfrak{Y}}_{m}\right)=\left\{d_{1} e^{* 0} \wedge \gamma ; \gamma \in \stackrel{p-2}{\wedge}\left(e^{* 1}, \ldots, e^{* 2 m}\right)\right\}
$$

Let $\psi: \wedge^{p-2}\left(e^{* 1}, \ldots, e^{* 2 m}\right) \rightarrow B^{p}\left(\mathfrak{E}_{m}\right), \quad \gamma \mapsto d_{1} e^{* 0} \wedge \ddot{\gamma} ;$ by the above result, $\psi$ is onto. By (i) and (ii), $\operatorname{dim} B^{p}\left(\mathfrak{S}_{m}\right)=\left(\begin{array}{c}2 m \\ p-2\end{array}\right)$; thus $\operatorname{dim} B^{p}\left(\tilde{\mathfrak{P}}_{m}\right)=$ $\operatorname{dim} \wedge^{p-2}\left(e^{* 1}, \ldots, e^{* 2 m}\right)$, which proves that $\psi$ is an isomorphism.

ACKNOWLEDGMENTS. This paper is Chapter IV of my thesis [7] achieved under the guidance of Professor M. P. Malliavin, to whom I am grateful. I must also thank Professor J. Dixmier for the reference [4, p. 197] which he pointed out in connection with (2.2). My friend, G. Favre, clarified some of my ideas about the Heisenberg Lie algebras and I am thankful to him. All my thanks to my colleague and friend, F. Du Cloux, for helpful conversations regarding spectral sequences. 


\section{REFERENCES}

1. C. Chevalley and S. Eilenberg, Cohomology theory of Lie groups and Lie algebras, Trans. Amer. Math. Soc. 63 (1948), 85- 124.

2. J. Dixmier, Cohomologie des algèbres de Lie nilpotentes, Acta Sci. Math. (Szeged) 16 (1955), 246-250.

3. S. I. Goldberg, On the Euler characteristic of a Lie algebra, Amer. Math. Monthly 62 (1955).

4. V. Guillemin and S. Sternberg, Geometric asymptotics, Math. Surveys, no. 14, Amer. Math. Soc., Providence, R.I., 1977.

5. G. Hochschild and J. P. Serre, Cohomologv of Lie algehras, Ann. of Math. (2) 57 (1953).

6. J. L. Koszul. Homologie et cohomologie des algèbres de Lie, Bull. Soc. Math. France 78 (1950), 65-127.

7. L. J. Santharoubane, Classification et cohomologie des algèbres de Lie nilpotentes, Thèse, Université de Paris 6. France. 1979.

Department of Mathematics, University of Poitiers, 86022 Poitiers, Cedex, France 